\title{
LIDAR system with electromagnetic two-axis scanning micromirror based on indirect time-of-flight method
}

\author{
Seung-Han Chung, Sung-Woo Lee, Seung-Ki Lee and Jae-Hyoung Park * (D)
}

\begin{abstract}
This paper presents a light detection and ranging (LIDAR) system using electromagnetically actuated two-axis scanning micromirror. The distance measurement with the LIDAR is based on the indirect time-of-flight method using the relative ratio of the accumulated charges in capacitors connected to photodiode pixels, which is determined by the time difference between the transmitted and reflected light pulse. The micromirror has double gimbaled structure for two-axis actuation and circular reflection plate with the diameter of $3 \mathrm{~mm}$. The horizontal scan angle of $49.13^{\circ}$ was obtained by the resonant actuation at $28 \mathrm{kHz}$, and the vertical scan of $29.23^{\circ}$ was achieved by the sinusoidal forced actuation at $60 \mathrm{~Hz}$. The distance to multiple targets could be measured at once by laser scanning using the micromirror, and the distance profile LIDAR image was constructed by the measurement results.
\end{abstract}

Keywords: LIDAR system, Micromirror, Indirect time-of-flight method, Distance measurement

\section{Introduction}

The LIDAR system has been one of the important research topics in the remote sensing technologies for three-dimensional imaging based on the distance measurement and diverse environmental monitoring. LIDAR has been studied intensively to be applied to the various applications such as autonomous vehicles, obstacle detection and security [1-5]. In the conventional LIDAR system, a motorized laser scanning unit such as galvanometer scanner is widely used for the detection of large area. In addition, LIDAR system has the disadvantages of having a large and heavy units with high cost. Recently, many research activities have been directed toward the development of LIDAR system using the micromirror, which has small size, light weight and low power consumption [6, 7].

In the LIDAR systems, the phase shift and time-of-flight method have been applied for the distance measurement. Laser beam is emitted to the target and reflected through receiving lens. In the phase shift method, the intensity

*Correspondence: parkjae@dankook.ac.kr

Department of Electronics and Electrical Engineering, Dankook University, Yongin 16890, South Korea modulated beam at a particular frequency is emitted to the target, and phase shift is produced in the reflected light depending on the distance to target [2, 8]. Even with the precise measurement of distance, however, the phase shift method requires a complicated system including laser beam modulation and data processing system. In addition, the phase shift method has disadvantage to be applied to real-time measurement because long processing time is required for precise distance ranging [ 1 , 9]. Time-of-flight method calculates the distance using time difference between transmitted and reflected light beam [10]. Time-of-flight method enables a simple system setup and distance calculation, but can make relatively large measurement error compared with the phase shift method [9]. The indirect time-of-flight calculates the distance to a target by measuring the phase difference between the emitted and the reflected pulse. Since the relative ratio of the phase difference determined by two pulse signals is used for the measurement of distance, the high-precision time measurement sensor is not required for the indirect time-of-flight method [11-14].

In this paper, we present the LIDAR system using electromagnetically actuated two-axis scanning micromirror based on the indirect time-of-flight method. The indirect 
time-of-flight method uses the relative ratio of the accumulated charges in capacitors connected to image sensor pixels, which is determined by the time difference between transmitted and reflected light beam. The distance measurement and imaging was demonstrated using the two-dimensional laser scanning with micromirror. Since the micromirror has a high scanning speed with small volume, it can make the system more compact and simple with high measurement rate compared to the system using conventional motor-based laser scanning units $[6,15,16]$.

\section{Design and experimental setup}

Figure 1 shows the schematic diagram of the LIDAR system using two-axis scanning micromirror for distance measurement. The LIDAR system is composed of a pulsed laser (Toptica laser beam, $640 \mathrm{~nm}, 150 \mathrm{~mW}$ ), scanning micromirror, receiving lens (TF $8 \mathrm{M}, 8 \mathrm{~mm}$ focal length, 1/3 inch), image sensor made of avalanche photodiode (Hamamatsu, S11963-01CR, $160 \times 120$ pixels), and data processing board. Figure 2 shows the LIDAR system setup on the optical table. The pulsed laser is transmitted and reflected at the two-axis scanning micromirror, and emitted to the targets. The laser is scanned to the objects through the micromirror. The beam scattered from the object enters the receiving lens and detected at the sensor. The data processing board converts the collected light information into the distance and image information. In the LIDAR system, the laser diode having $640 \mathrm{~nm}$ wavelength and $150 \mathrm{~mW}$ average power was used as light emitting source with the adjustable pulse duration from $3 \mathrm{~ns}$ to continuous wave. The image sensor with

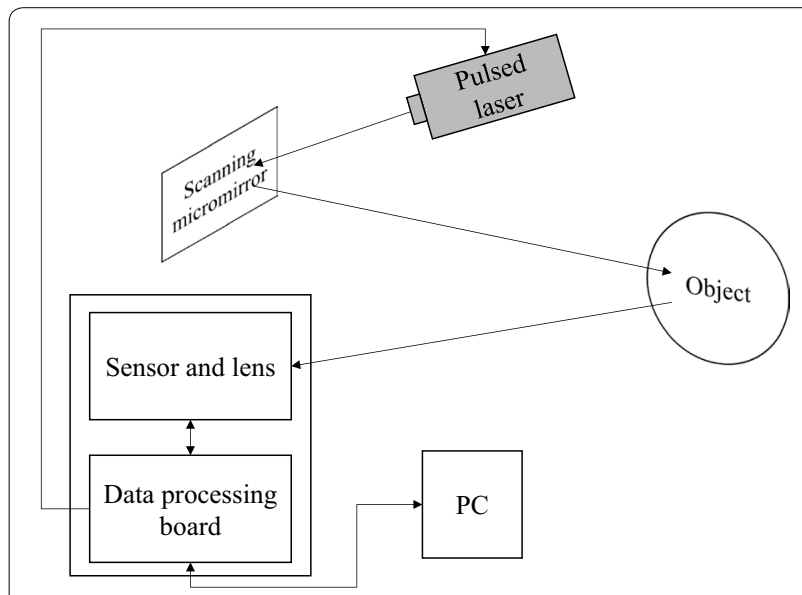

Fig. 1 Schematic diagram of the LIDAR system using two-axis scanning micromirror

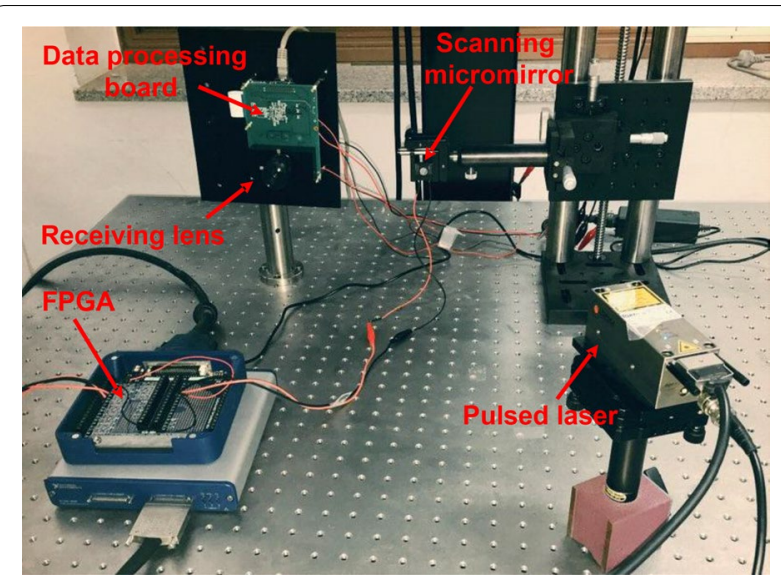

Fig. 2 LIDAR system setup on the optical table

$160 \times 120$ pixels photodiode array and receiving lens with the field of view of $37.5^{\circ} \times 27.7^{\circ}$ were used.

Figure 3 shows the basic circuit structure at the photosensitive area of the photodiode to obtain the distance with indirect time-of-flight method. The distance measurement is using the relative ratio of the accumulated charges in each capacitor connected to photodiode output. The pulse timing chart at the circuit is shown in Fig. 4. The transmitted and reflected light pulses are shown. $\mathrm{T}_{0}$ is transmitted light pulse width, and $\mathrm{T}_{\mathrm{d}}$ is time difference between transmitted and reflected light pulse, which is dependent on the distance from source to target. The pulses to turn on the switch 1 and 2 are also shown, which are connected to the capacitor $1\left(\mathrm{C}_{1}\right)$ and $2\left(C_{2}\right)$, respectively. The switch 1 turn-on pulse is synchronously generated with the transmitted light pulse, and then the switch 2 pulse is generated at the end of switch 1 pulse. Therefore, $Q_{1}$ represents the amount of charge accumulated in $\mathrm{C}_{1}$ during the switch 1 turn-on period, which is generated at the photodiode due to the reflected light pulse. $Q_{2}$ is the accumulated charge in $C_{2}$ from the reflected light pulse. The ratio of the charge is determined by the time difference between the transmitted and reflected light pulse. Therefore, the distance D is calculated by Eq. (1);

$$
D=\frac{1}{2} \times c \times T_{0} \times \frac{Q_{2}}{Q_{1}+Q_{2}}
$$

where $\mathrm{c}$ is light velocity, $T_{0}$ is transmitted light pulse width, $Q_{1}$ and $Q_{2}$ are charges accumulated in $C_{1}$ and $\mathrm{C}_{2}$, respectively. The switch 3 is to discharge unneeded charges caused by ambient light during the non-emission period. In the experiment, the switch 3 turn-on pulse width was set to $40 \mathrm{~ns}$. The image sensor signals from reflected light and charge-to-voltage conversion 


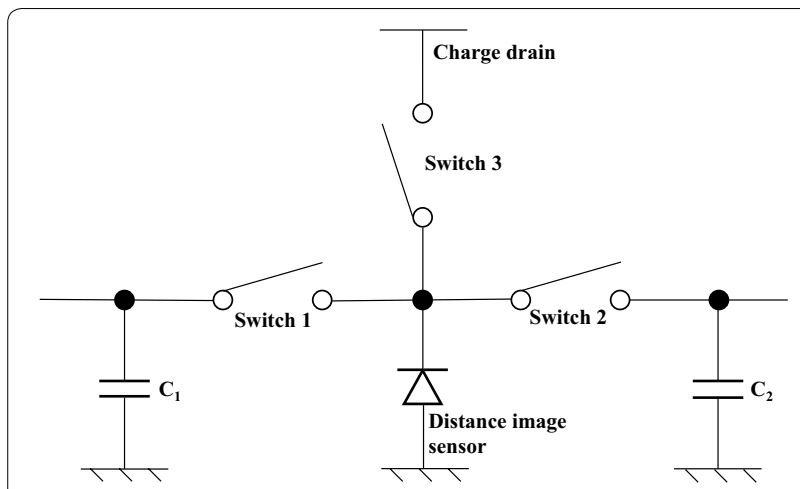

Fig. 3 Indirect time-of-flight circuit structure at the photosensitive area of the photodiode

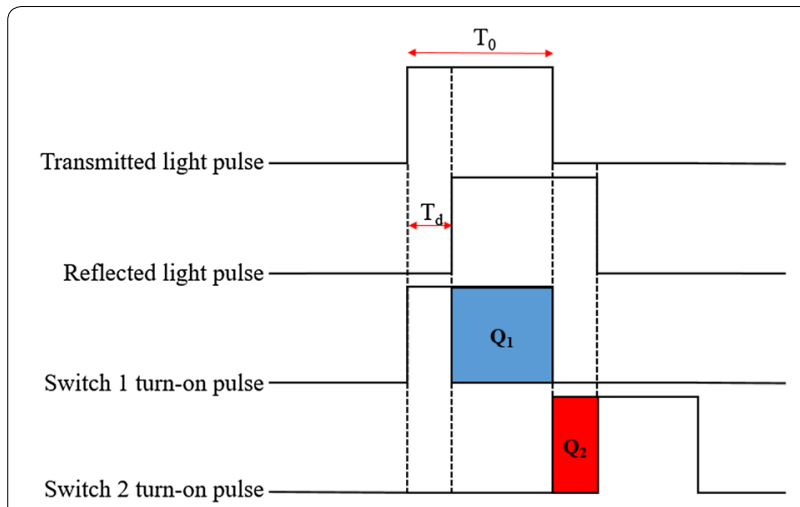

Fig. 4 Pulse timing chart for the distance measurement of indirect time-of-flight method

on accumulated charges are processed to calculate distance through the data processing board (S11963-01CR, Hamamatsu) [17]. In the LIDAR system, the transmitted light pulse width $\left(\mathrm{T}_{0}\right)$ was set to $90 \mathrm{~ns}$, which means the measureable distance to an object is $13.5 \mathrm{~m}$.

\section{Electromagnetic two-axis scanning micromirror}

The electromagnetically actuated two-axis scanning micromirror was employed in the LIDAR system, which was developed in our previous study [18]. The scanning micromirror used in the LIDAR system is shown in Fig. 5. The micromirror device consists of a microfabricated double gimbaled structure and a set of permanent magnets for high magnetic field generation. A current path using electroplated copper coil is formed on the backside to generate torque under magnetic field. The size of the circular mirror plate is $3 \mathrm{~mm}$ in diameter, on which an aluminum reflective surface is formed. The fast horizontal scan is obtained by the resonant mode actuation, while the tilting for slow vertical scan is achieved by forced actuation. The micromirror is assembled with permanent

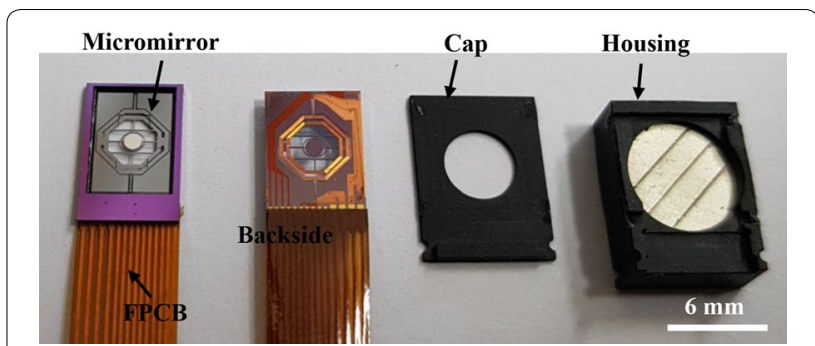

Fig. 5 Two-axis scanning micromirror and assembly housing with permanent magnets
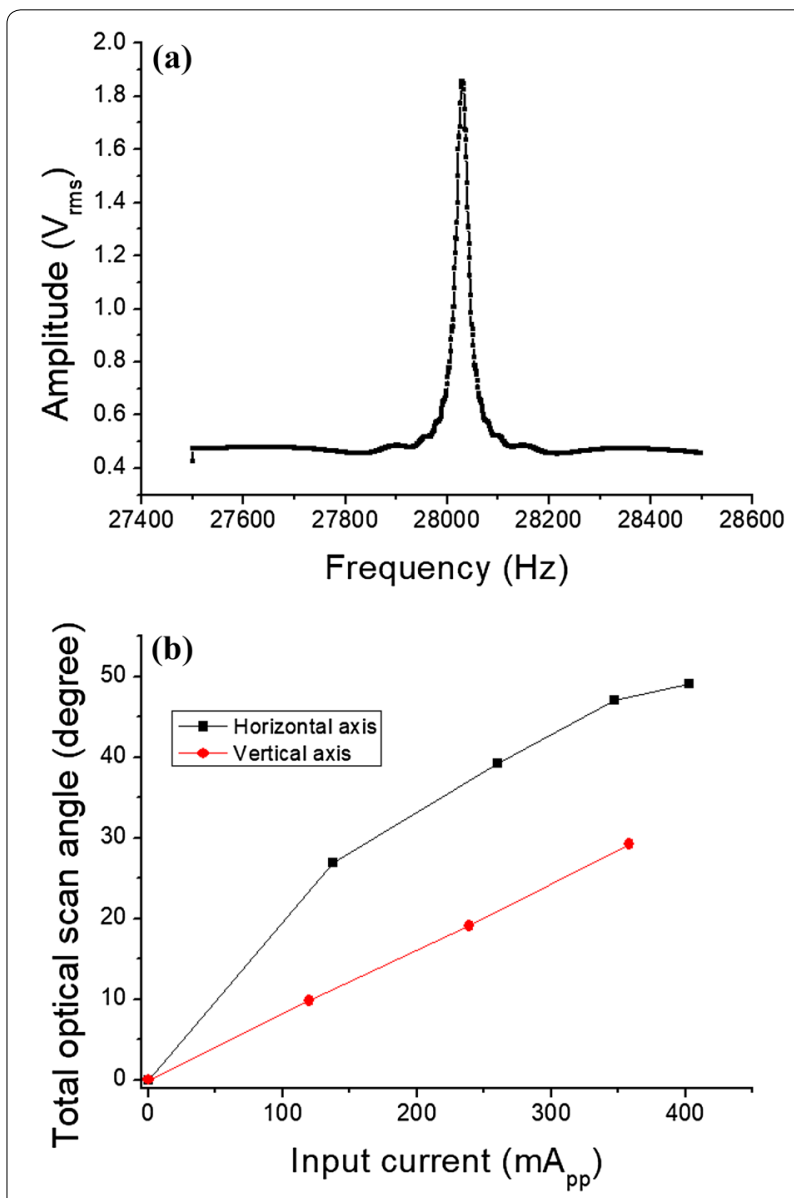

Fig. 6 a Frequency response of the micromirror for the horizontal scan, b Measured scan angle

magnets using plastic housing, and the volume of the packaged device is $6.6 \mathrm{~mm} \times 11 \mathrm{~mm} \times 4.72 \mathrm{~mm}$. For horizontal scan, maximum optical scan angle of $49.13^{\circ}$ at the resonance frequency of $28.0 \mathrm{kHz}$ was obtained. The vertical scan angle was $29.23^{\circ}$ at $60 \mathrm{~Hz}$ as shown in Fig. 6. 

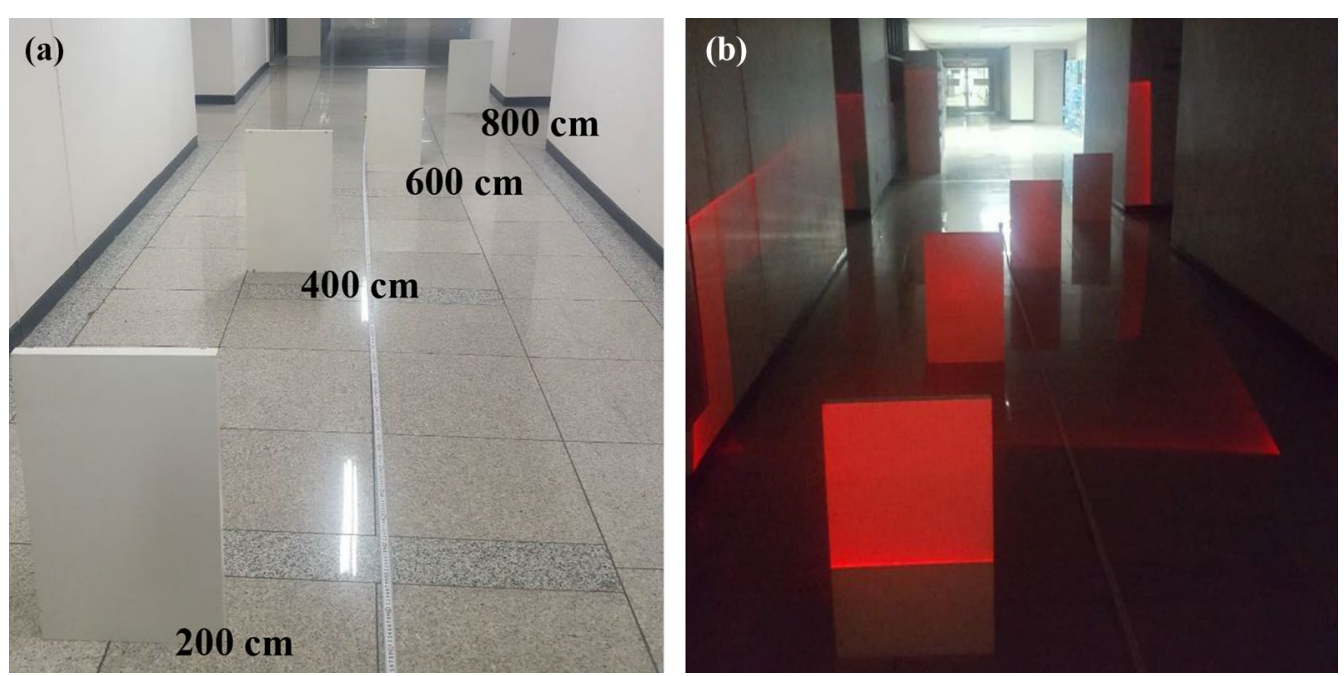

Fig. 7 a Target object setup to test the distance measurement using the LIDAR system, b laser scan image to the targets using two-axis scanning micromirror

\section{LIDAR image and distance measurements}

Figure 7a shows target object setup to test the distance measurement using the LIDAR system. White panels were used for the targets, which are placed at 200, 400, 600 , and $800 \mathrm{~cm}$ from the laser. The laser scan image to the targets using the two-axis scanning micromirror is shown in Fig. 7b. All the targets are in the field of view of micromirror optical scan angle, and the distance to multiple targets were measured at the same time with the laser scan. The experiment was performed in the dark environment, where the ambient light was blocked.

Figure $8 \mathrm{a}$ shows the distance profile image in the scanned area, which is constructed by the distance measurement results at each pixel of photodiode image sensor. At each pixel, the distance was obtained by the average value of calculation using the charge ratio of $\mathrm{Q}_{1}$ and $\mathrm{Q}_{2}$ at a frame rate of $100 / \mathrm{s}$. In addition, the distance values of $15 \times 15$ pixels were taken at the center of each target panel and averaged to determine the measured distance to each target. Figure $8 \mathrm{~b}$ shows the comparison of the measured distance with the actual distance to each target. The measurement distances are the average values for 100 frame. For 200, 400, 600, and $800 \mathrm{~cm}$ targets, the distance was measured to be $204.8 \pm 17.8,404.7 \pm$ $37.6,625.4 \pm 23.8$, and $840.6 \pm 12.1 \mathrm{~cm}$, respectively. The measurement errors tend to increase as increasing the distance. The distance resolution of the system is obtained to be about $36 \mathrm{~cm}$ using 3 standard deviation rule, which is the general method to calculate the limit of detection. It is considered that the main factor related to (a)

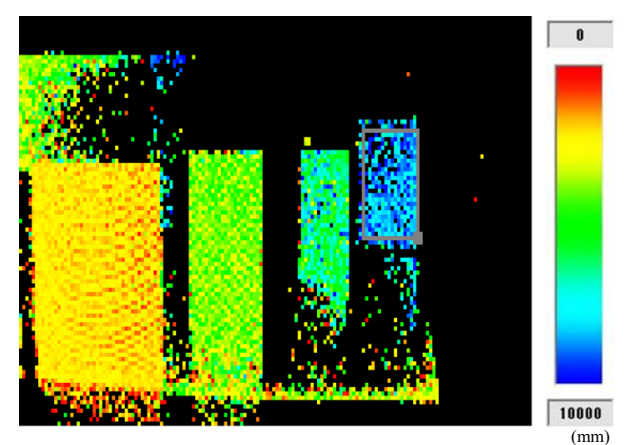

(b)

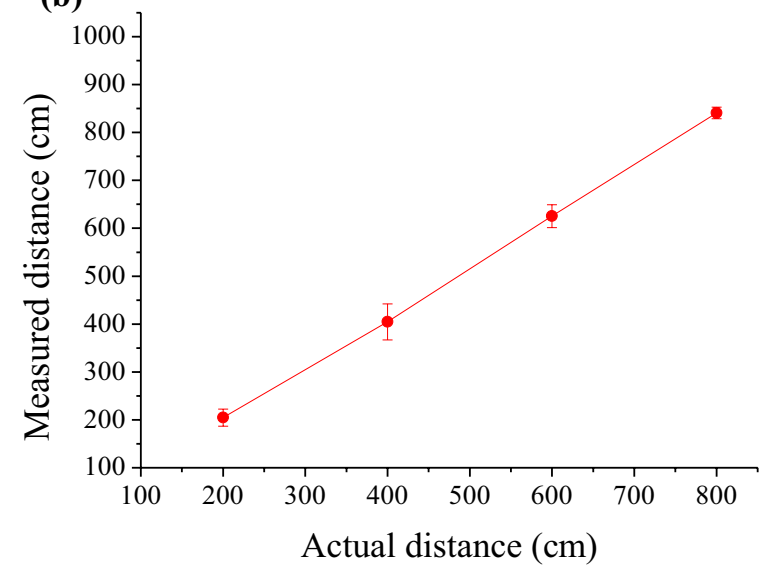

Fig. 8 a Distance profile image in the scanned area, $\mathbf{b}$ comparison of the measured distance with the actual distance to each target 
distance resolution is synchronization of the pulsed laser with the control signal. Laser diode is controlled and synchronized by the pulse signal from the processing board. The control signal pulse width was set to $90 \mathrm{~ns}$, but the laser pulse synchronization error was about 2 ns. The results show that two-axis scanning micromirror could be applied for the LIDAR system based on the indirect time-of-flight method with the distance measurement.

\section{Conclusion}

In this paper, the feasibility of the LIDAR system using electromagnetic two-axis scanning micromirror has been successfully demonstrated based on indirect timeof-flight method. By using the transmitted laser scan with the micromirror and the ratio of the accumulated charges in capacitors determined by the reflected light, the distance to multiple targets could be measured at the same time, and the distance profile LIDAR image could be obtained. The distance measurement results show that the scanning micromirror can be readily applied to the indirect time-of-flight LIDAR system. The proposed LIDAR system is expected to be used in various application areas with further improvement of the LIDAR optics combined with the scanning micromirror.

\section{Authors' contributions}

JHP devised the idea and supervised the project. JHP, SHC, SWL, and SKL discussed the design and experimental setup. SHC performed the LIDAR experiment using the scanning micromirror. JHP and SHC drafted the manuscript. All authors read and approved the final manuscript.

\section{Acknowledgements}

Not applicable.

\section{Competing interests}

The authors declare that they have no competing interests.

\section{Availability of data and materials}

Not applicable.

\section{Funding}

This research was supported by the Space Core Technology Development Program through the National Research Foundation of Korea (NRF) funded by the Ministry of Science and ICT (NRF-2013M1A3A3A02042410).

\section{Publisher's Note}

Springer Nature remains neutral with regard to jurisdictional claims in published maps and institutional affiliations.

Received: 12 December 2018 Accepted: 8 March 2019

Published online: 12 March 2019

\section{References}

1. Hu P, Tan J, Yang H, Zhao X, Liu S (2011) Phase-shift laser range finder based on high speed and high precision phase-measuring techniques.
In: Proceedings of the 10th international symposium of measurement technology and intelligent instrument, Daejeon, 1-5 July 2011

2. Lefsky MA, Cohen WB, Parker GG, Harding DJ (2002) Lidar remote sensing for ecosystem studies: Lidar, an emerging remote sensing technology that directly measures the three dimensional distribution of plant conopies, can accurately estimate vegetation structural attributes and should be of particular interest to forest, landscape, and global ecologists. Bioscience 52:19-30

3. Asvadi A, Premebida C, Peixoto P, Nunes U (2016) 3D Lidar-based static and moving obstacle detection in driving environments: an approach based on voxels and multi-region ground planes. Rob Auton Syst 83:299-311

4. Gietelink O, Ploeg J, Schutter BD, Verhaegen M (2006) Development of advanced driver assistance systems with vehicle hardware-in-the-loop simulations. Vehicle Syst Dyn 44:569-590

5. Takai I, Matsubara H, Soga M, Ohta M, Ogawa M, Yamashita T (2016) Single-photon avalanche diode with enhanced NIR-sensitivity for automotive LIDAR Systems. Sensors 16:459-467

6. Niclass C, Ito K, Soga M, Matsubara H, Aoyagi I, Kato S, Kagami M (2012) Design and characterization of a 256x64-pixel single-photon imager in CMOS for a MEMS-based laser scanning time-of-flight sensor. Opt Express 20:11863-11881

7. Brigante CMN, Abbate N, Basile A, Faulisi AC, Sessa S (2011) Towards miniaturization of a MEMS-based wearable motion capture system. IEEE Trans Ind Electron 58:3234-3241

8. Bamji CS, O'Connor P, Elkhatib T, Mehta S, Thompson B, Prather LA, Snow D, Akkaya OC, Daniel A, Payne AD, Perry T, Fenton M, Chan VH (2015) A $0.13 \mu \mathrm{m}$ CMOS system-on-chip for a $512 \times 424$ time-of-flight image sensor with multi-frequency photo-demodulation up to $130 \mathrm{MHz}$ and $2 \mathrm{GS} / \mathrm{s}$ ADC. IEEE J Solid-State Circuits 50:303-319

9. Amann M-C, Bosch TM, Lescure M, Myllylae RA, Rioux M (2001) Laser ranging: a critical review of unusual techniques for distance measurement. Opt Eng 40:10-19

10. Gokturk SB, Yalcin H, Bamji C (2004) A time-of-flight depth sensor-system description, issues and solutions. In: Proceedings of the 4th IEEE Computer vision and pattern recognition workshop, Washington DC, 35-43 May 2006

11. Perenzoni M, Stoppa D (2011) Figures of merit for indirect time-of-flight $3 \mathrm{D}$ cameras: definition and experimental evaluation. Remote Sens 3:2461-2472

12. Bellisai S, Villa F, Tisa S, Bronzi D (2012) Indirect time-of-flight 3D ranging based on SPADs. In: Proceedings of quantum sensing and nanophotonics devices IX, California, Jan 2012

13. Jang J, Hwang S, Park K (2013) Design of indirect time-of-flight based lidar for precise three-dimensional measurement under various reflection conditions. In: Proceedings of the 4th international conference on sensor device technologies and applications, Barcelona, 25-29 Aug 2013

14. Yasutomi K, Usui T, Han S-M, Takasawa T, Kagawa K, Kawahito S (2014) An indirect time-of-flight measurement technique with impulse photocurrent response for sub-millimeter range resolved imaging. Opt Express 22:18904-18913

15. Kasturi A, Milanovic V, Atwood BH, Yang J (2016) UAV-borne lidar with MEMS mirror-based scanning capability. In: Proceedings of the laser radar technology and applications XXI, Maryland, May 2016

16. Hu Q, Pedersen C, Rodrigo PJ (2016) Eye-safe diode laser doppler lidar with a MEMS beam-scanner. Opt Express 24:1934-1942

17. http://www.hamamatsu.com/us/en/product/category/3100/4005/4148/ S11963-01CR/index.html. Accessed 02 Feb 2018

18. Ju S, Jeong H, Park J-H, Ji C-H (2018) Electromagnetic 2D scanning micromirror for high definition laser projection displays. IEEE Photonic Tech Lett 30:2072-2075 